\title{
Domiciliary nocturnal nasal intermittent positive pressure ventilation in hypercapnic respiratory failure due to chronic obstructive lung disease: effects on sleep and quality of life
}

\author{
M W Elliott, A K Simonds, M P Carroll, J A Wedzicha, M A Branthwaite
}

\begin{abstract}
Background Domiciliary assisted ventilation, using negative or positive pressure devices, is an effective treatment for respiratory failure due to chest wall deformity and neuromuscular disease. Negative pressure ventilators have been used with some success in patients with chronic obstructive lung disease in hospital, but attempts to continue treatment at home have been disappointing. This study evaluates the practicalities of nasal intermittent positive pressure ventilation at home in patients with chronic obstructive lung disease and the effect on sleep and quality of life.
\end{abstract}

Methods and results Twelve patients with chronic obstructive lung disease and hypercapnic respiratory failure received nasal intermittent positive pressure ventilation at home during sleep. At six months eight were continuing with the ventilation. One patient had died and three had withdrawn because they were unable to sleep with the equipment. Full polysomnography performed during ventilation in patients continuing treatment at six months showed an increase in mean $\mathrm{PaO}_{2}$ of $11 \%(+2 \%$ to $+23 \%)$ and lower mean transcutaneous carbon dioxide tensions (by $-2 \cdot 7(-1.3$ to $-5 \cdot 1) \mathrm{kPa}$ ) overnight compared with spontaneous breathing before the start of nasal intermittent positive pressure ventilation. Total sleep time and sleep efficiency changed during ventilation by $+72 \cdot 5(+21$ to +204$)$ minutes and $+5 \%(-3 \%$ to $+30 \%)$ respectively; sleep architecture and the number of arousals were unchanged. Quality of life did not change but was no worse during ventilation. At one year seven patients were still using the ventilator and $\mathrm{PaCO}_{2}$ and bicarbonate ion concentration during the day had improved further by comparison with the values at six months (change from baseline $-1.7(-2.1$ to -0.6$) \mathrm{kPa}, \mathrm{p}<0.05$, and $-6.3(-11.9$ to -4$) \mathrm{mmol} / \mathrm{l}$, p $<0.05$ ).

Conclusions Nasal intermittent positive pressure ventilation can be used effectively at home during sleep in selected patients with chronic obstructive lung disease. Its future place in management can be established only by formal comparison with long term oxygen therapy.
Nasal intermittent positive pressure ventilation is becoming increasingly recognised as an effective method of supporting patients with respiratory failure due to neuromuscular disease, chest wall deformity, and to a lesser extent chronic obstructive lung disease.$^{1-3}$ It requires no cumbersome external equipment, maintains the patency of the upper airway, and has been used effectively at home overnight.

We have previously reported improved daytime arterial blood gas tensions during spontaneous breathing by day after six months in patients using nasal intermittent positive pressure ventilation regularly at night. ${ }^{4}$ The possible mechanisms of these improvements were discussed. In this paper we have looked at the feasibility of domiciliary nasal intermittent positive pressure ventilation overnight in patients with chronic obstructive lung disease, the extent to which nocturnal hypoventilation is controlled, and the resulting effect on sleep and quality of life. Follow up has been continued for one year and arterial blood gas tensions at the end of the study are reported.

\section{Methods}

PATIENTS

The study was approved by the ethics committee of the Royal Brompton and National Heart Hospital, and all patients gave informed consent.

Nine male and three female patients with severe stable chronic obstructive lung disease were equipped with domiciliary ventilators. Their clinical details are given in table 1. All had severe airflow limitation and were hypoxaemic and hypercapnic at the time of recruitment. All had past or present peripheral oedema and therefore met the criteria for domiciliary oxygen therapy. Nine patients were recruited from outpatient clinics and their clinical stability was confirmed by a variation of less than $5 \%$ in arterial blood gas tensions and forced expiratory volume in one second $\left(\mathrm{FEV}_{1}\right)$ at the beginning and end of a four week run in period. One patient (case 7) was first seen as an inpatient but did not start nasal intermittent positive pressure ventilation until six weeks after discharge.

The two other patients (cases 8 and 11) had a long history of progressive deterioration despite maximum conventional treatment, including oxygen, and had been referred with severe 
Table 1 Clinical details on patients before starting nasal intermittent positive pressure

\begin{tabular}{|c|c|c|c|c|c|c|c|c|c|}
\hline Case No & $\begin{array}{l}\text { Age } \\
\text { (years), } \\
\text { sex }\end{array}$ & $\begin{array}{l}F E V_{1} \\
(1)\end{array}$ & $\begin{array}{l}F E V \mid F V C \\
(\%)\end{array}$ & $\begin{array}{l}R V \\
(1)\end{array}$ & $\begin{array}{l}T L C \\
(1)\end{array}$ & $\begin{array}{l}\text { Kco (mmol/ } \\
\min / \mathrm{kPa} / \mathrm{l})\end{array}$ & $\begin{array}{l}\mathrm{PaO}_{2} \\
(\mathrm{kPa})\end{array}$ & $\begin{array}{l}\mathrm{PaCO}_{2} \\
(\mathrm{kPa})\end{array}$ & $\begin{array}{l}\mathrm{HCO}_{3}^{-} \\
(\mathrm{mmol} / \mathrm{l})\end{array}$ \\
\hline $1^{\star}$ & $55, M$ & 0.88 & 38 & 2.74 & 5.44 & 1.47 & 5.9 & 8.0 & 36.9 \\
\hline $2^{\star}$ & $59, \mathrm{M}$ & 0.86 & 44 & 3.44 & 5.44 & 1.84 & 6.3 & $.7 \cdot 6$ & 35.5 \\
\hline $3 \star$ & $64, M$ & $1 \cdot 10$ & 39 & $4 \cdot 20$ & $7 \cdot 70$ & 1.49 & 6.7 & $7 \cdot 1$ & $34 \cdot 4$ \\
\hline 4 & 58, M & 0.56 & 26 & 4.95 & $7 \cdot 30$ & 0.87 & 6.5 & $8 \cdot 1$ & $35 \cdot 5$ \\
\hline $5^{\star}$ & $50, M$ & 0.57 & 32 & $3 \cdot 12$ & 5.02 & $1 \cdot 18$ & 6.9 & 6.3 & 31.7 \\
\hline $6^{\star}$ & 53, M & 0.44 & 28 & 3.76 & 5.66 & 1.44 & $6 \cdot 4$ & 7.9 & $37 \cdot 4$ \\
\hline 7 & $64, M$ & 0.61 & 30 & $4 \cdot 52$ & $7 \cdot 27$ & 0.57 & $6 \cdot 8$ & $8 \cdot 3$ & $37 \cdot 6$ \\
\hline $8^{\star}$ & $49, F$ & 0.48 & 35 & $2 \cdot 43$ & $4 \cdot 13$ & 1.27 & $7 \cdot 0$ & $7 \cdot 3$ & $34 \cdot 0$ \\
\hline 9 & $62, F$ & 0.26 & 23 & $4 \cdot 40$ & $5 \cdot 55$ & 0.63 & 5.9 & $8 \cdot 1$ & $40 \cdot 0$ \\
\hline 10 & $62, M$ & 0.59 & 30 & 2.91 & 4.81 & 1.77 & 6.5 & 6.8 & 31.8 \\
\hline $11^{\star}$ & $51, \mathrm{M}$ & 0.51 & 19 & 4.64 & $7 \cdot 34$ & $1 \cdot 11$ & $5 \cdot 6$ & 9.9 & $40 \cdot 0$ \\
\hline 12 & $62, F$ & 0.29 & 30 & 2.97 & $4 \cdot 17$ & 1.57 & 6.9 & $7 \cdot 0$ & 33.0 \\
\hline Mean (SD) & $57 \cdot 4(5 \cdot 6)$ & $0.60(0.25)$ & $31(7)$ & $3.67(0.85)$ & $5.82(1.27)$ & $1.27(0.41)$ & $6.5(0.5)$ & $7.7(0.9)$ & $35.7(2.9)$ \\
\hline
\end{tabular}

FEV - forced expiratory volume in one second; FVC_forced vital capacity; RV-residual volume; TLC_total lung capacity; $\mathrm{KcO}-$ carbon monoxide transfer coefficient; $\mathrm{PaO}_{2}$ - arterial oxygen tension; $\mathrm{PaCO}_{2}$-arterial carbon dioxide tension; [ $\left.\mathrm{HCO}_{3}^{-}\right]-$ bicarbonate concentration.

$\star$ Using domiciliary oxygen.

symptomatic respiratory failure (arterial oxygen tension $\left(\mathrm{PaO}_{2}\right) 3.5 \mathrm{kPa}$, arterial carbon dioxide tension $\left(\mathrm{PaCO}_{2}\right) \mathbf{8 . 4} \mathrm{kPa}$ and $\mathrm{PaO}_{2}$ $4.9 \mathrm{kPa}, \mathrm{PaCO}_{2} 12.9 \mathrm{kPa}$ respectively). They were resuscitated by nasal intermittent positive pressure ventilation and discharged after 17 and 12 days of assisted ventilation as inpatients with noticeably improved blood gas tensions $\left(\mathrm{PaO}_{2} 7 \cdot 1 \mathrm{kPa}, \mathrm{PaCO}_{2} 8 \cdot 2 \mathrm{kPa}\right.$ and $\mathrm{PaO}_{2} 7.9 \mathrm{kPa}$, $\mathrm{PaCO}_{2} 6.6 \mathrm{kPa}$ respectively). Although we had intended to delay these patients' recruitment for six weeks, domiciliary ventilation was started earlier, at the patients' request, because of clinical deterioration. At the time of recruitment arterial blood gas tensions were better than when they first presented to this hospital. Patient 8 was taking protriptyline $10 \mathrm{mg}$ daily, ${ }^{56}$ which was discontinued, and prednisolone $30 \mathrm{mg}$ daily, which was reduced to $2.5 \mathrm{mg}$ daily by six months. Patient 11 was taking prednisolone $20 \mathrm{mg}$ daily at the time of recruitment, which was discontinued.

In the remainder of the patients treatment was unchanged over the trial period with the exceptions of patients 1 (inhaled high dose beclomethasone stopped), 6 (prednisolone increased from $12.5 \mathrm{mg}$ to $15 \mathrm{mg}$ daily and frusemide decreased from $120 \mathrm{mg}$ to $40 \mathrm{mg}$ daily), and 7 (diuretic treatment reduced from bumetanide $5 \mathrm{mg}$, metolazone $5 \mathrm{mg}$, and amiloride $10 \mathrm{mg}$ to bumetanide $1 \mathrm{mg}$ and amiloride $5 \mathrm{mg}$ daily). Patients also reported a reduction in requirements for inhaled $\beta_{2}$ agonists, which had been prescribed to be taken as required. Seven patients were using domiciliary oxygen therapy up to the time of starting nasal intermittent positive pressure ventilation but agreed to discontinue it for the duration of the study.

Patients were acclimatised to nasal intermittent positive pressure ventilation in hospital with the Bromptonpac ventilator (Pneupac, Luton) and a commercially available nasal mask (Respironics, Monroeville, Pennsylvania). The Bromptonpac is a volume cycled flow generator capable of delivering large minute volumes at high pressure. All patients were ventilated with air, and ventilator settings were adjusted to the patients' comfort and to maxi- mise gas exchange. The patients in cases 1-4 were equipped with the ventilator, instructed in its use, and discharged on the same day. Three out of four had difficulty acclimatising to the technique so subsequent patients remained in hospital until they were confident in using the equipment. This entailed a median stay in hospital of three (range 2 to 10) days. Patients were readmitted after four weeks of using the ventilator at home for overnight monitoring of oxygen saturation $\left(\mathrm{SaO}_{2}\right)$ and transcutaneous $\mathrm{CO}_{2}\left(\mathrm{PtcCO}_{2}\right)$; small adjustments were made to ventilator settings if necessary.

\section{PROTOCOL}

Arterial blood gas tensions and quality of life were measured before the start of nasal intermittent positive pressure ventilation and after six months of home use in all patients. In addition full polysomnography was performed before the start of nasal intermittent positive pressure ventilation and was repeated during ventilation after six months in patients still using the equipment. Arterial blood gas tensions were measured again after one year.

\section{INVESTIGATIONS}

After a night of acclimatisation full polysomnography was performed in a sleep laboratory. An electroencephalogram and electro-oculograms (EOG) were measured with silver cup electrodes and standard lead placements. ${ }^{7}$ A submental electromyogram was measured with surface electrodes. Movements of the chest and abdominal wall were monitored with two pairs of magnetometers positioned at the widest anteroposterior diameter of chest and abdomen respectively. The magnetometers were not calibrated for volume so provided only qualitative data on ventilation. Oronasal airflow was monitored with thermistors positioned over the lower lip and in the nasal vestibule. Magnetometer and airflow signals were processed with custom built amplifiers.

$\mathrm{SaO}_{2}$ was measured continuously at the earlobe with an Ohmeda Biox III pulse oximeter (Ohmeda, Louisville, Colorado) and $\mathrm{PtcCO}_{2}$ by a Hewlett Packard capnometer (Hewlett Pack- 
ard, Waltham, Massachusetts) with the sensor placed on the forearm. All signals were stored on a Neuroscience Neuromapper (Neuroscience, Harold Hill, Essex). Studies started at 2300 and continued for eight hours or until the patient awoke and wanted to get up. The studies performed during nasal intermittent positive pressure ventilation were exactly the same as those during spontaneous ventilation except that measurement of oronasal airflow was replaced by that of mask pressure, with a catheter taken from an integral port in the nasal mask and connected to a pressure transducer.

Sleep was staged manually in 30 second epochs according to the criteria of Rechtschaffen and Kales. ${ }^{7}$ The following variables were analysed:

- Time in bed-the time from lights out to the last awakening

- Wakefulness (stages wake and I) and rapid eye movement (REM) and non-REM sleep (stages II, III, and IV)

- Total sleep time-the time in bed minus periods of wakefulness

- Sleep efficiency-total sleep time as a percentage of the time in bed

- Sleep latency and the number of arousals (an arousal was defined as a return to stage wake).

A rolling mean oxygen saturation and $\mathrm{PtcCO}_{2}$ was determined by the computer, time as well as magnitude of change thus being taken into
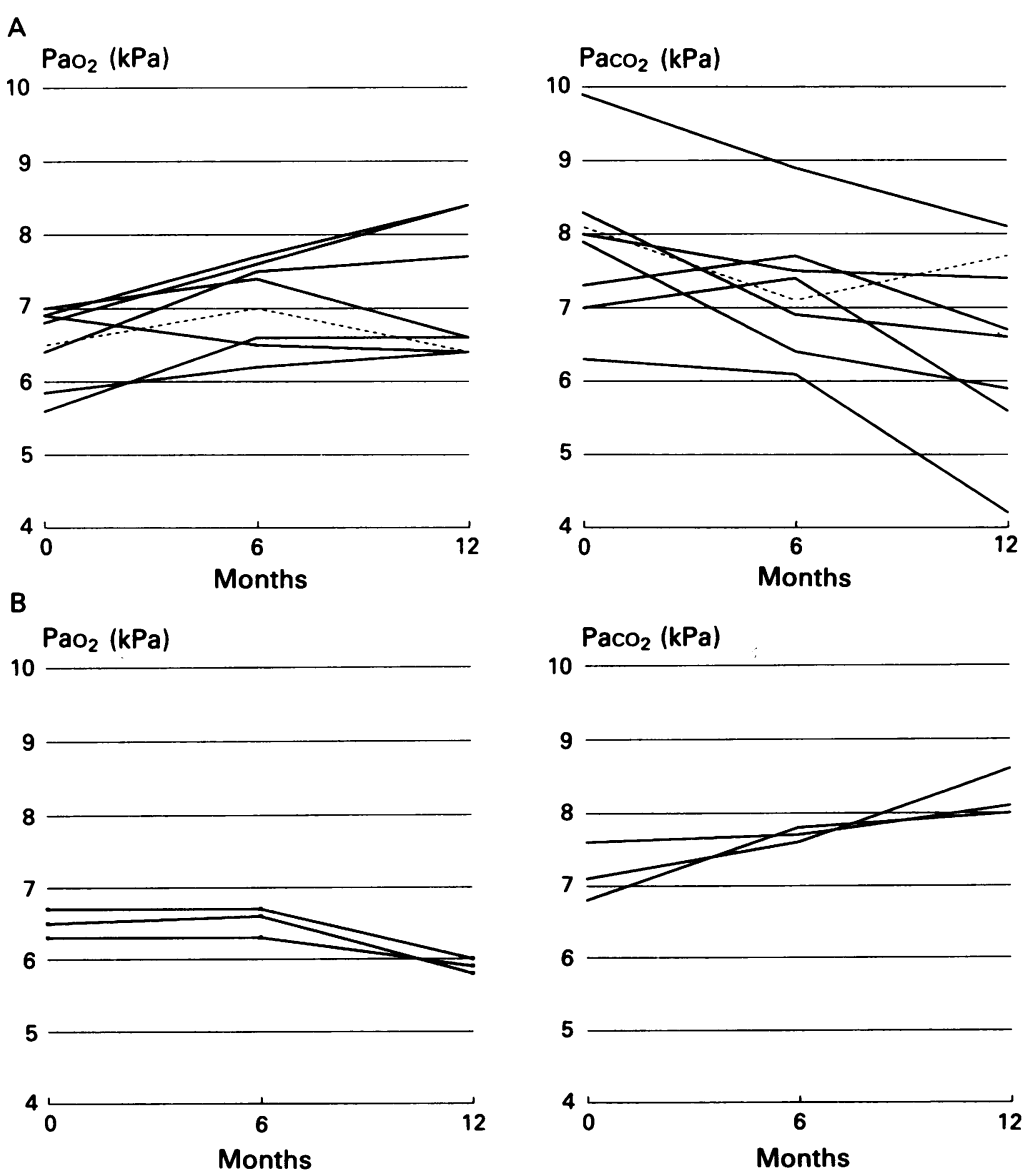

Figure 1 Arterial blood gas tensions during spontaneous breathing in the mid afternoon before starting nasal intermittent positive pressure ventilation and after six months and one year. A: Seven patients still using ventilation at home at one year (solid lines) and one patient (case 4) who discontinued ventilation after nine months (broken line).

$B$ : Three patients who discontinued home ventilation before six months. consideration. Mean and minimum $\mathrm{SaO}_{2}$ and extent of desaturation from baseline were determined during REM and non-REM sleep and wakefulness. The mean $\mathrm{PtcCO}_{2}$ overnight and the maximum increment in $\mathbf{P t c C O}_{2}$ from baseline values during wakefulness were also determined.

Daytime arterial blood gas tensions were measured in the mid afternoon when the patient had been sitting quietly for at least 15 minutes breathing room air. Samples were processed by a Corning 178 blood gas analyser (Ciba-Corning, Halstead, Essex).

Quality of life was assessed with a standard questionnaire developed for use in patients with chronic obstructive lung disease. ${ }^{8}$ At the second visit patients were not allowed to see their previous responses.

\section{STATISTICAL ANALYSIS}

Statistical analysis of all data was performed with the Minitab statistical package (Minitab Inc, State College, Pennsylvania) and the Wilcoxon signed rank test, with the level of significance set at $p<0.05$. Data are expressed as medians and ranges, unless otherwise stated.

\section{Results}

Eight patients completed six months' domiciliary nasal intermittent positive pressure ventilation. One patient (case 9) died after six weeks of worsening respiratory failure. She had very severe airflow obstruction and limited exercise tolerance and, although enthusiastic to join the trial, never totally mastered the technique and was not able to use it satisfactorily at home. Three patients voluntarily discontinued home ventilation, two within three months (cases 2 and 3 ) and one after five months (case 10). All three had had relatively undisturbed sleep before starting nasal intermittent positive pressure ventilation - two of them while using oxygen at home (cases 2 and 3)-but were unable to sleep so well with the ventilator. They had less airflow obstruction (table 1) and less hypercapnia (fig 1) than the patients who continued domiciliary nasal intermittent positive pressure ventilation. The ventilator was withdrawn from one patient (case 4), whose compliance was poor, after nine months because of worsening cor pulmonale; he was transferred to domiciliary oxygen therapy but continued to deteriorate and required several admissions to hospital. Seven patients were still using the equipment at one year. The patients who were treated successfully used the machine for between six and 10 hours each night.

$\mathrm{SaO}_{2}$ during sleep and the quality of sleep during spontaneous ventilation before and after six months of nasal intermittent positive pressure ventilation are shown in tables 2 and 3 . An example of overnight oxygen saturation and $\mathrm{PtcCO}_{2}$ during spontaneous ventilation and nasal intermittent positive pressure ventilation is shown in figure 2. There was no evidence that any of the patients had obstructive sleep apnoea. In the patients continuing treatment at six months mean oxygen saturation was $11 \%$ 
Table 2 Mean (SD) average and minimum oxygen saturation ( $\mathrm{SaO}_{2}$ ) and average and maximum oxygen desaturation from waking values during wakefulness, REM and non-REM (NREM) sleep during spontaneous breathing $(S V)$ at the study onset and during nasal intermittent positive pressure ventilation (NIPPV) six months later

\begin{tabular}{|c|c|c|c|c|c|c|}
\hline & \multicolumn{3}{|c|}{ Average $\mathrm{SaO}_{2}(\%)$} & \multicolumn{3}{|c|}{ Minimum $\mathrm{SaO}_{2}(\%)$} \\
\hline & $S V$ & $p$ & NIPPV & $S V$ & $p$ & NIPPV \\
\hline \multirow[t]{2}{*}{$\begin{array}{l}\text { WAKE } \\
\text { NREM } \\
\text { REM }\end{array}$} & $\begin{array}{l}81(6.0) \\
77(8.9) \\
69(11 \cdot 4)\end{array}$ & $\begin{array}{l}<0.05 \\
<0.05 \\
<0.05\end{array}$ & $\begin{array}{l}91(2.9) \\
89(4 \cdot 6) \\
85(9 \cdot 3)\end{array}$ & $\begin{array}{l}70(9 \cdot 0) \\
62(11 \cdot 4) \\
55(10 \cdot 7)\end{array}$ & $\begin{array}{l}\text { NS } \\
\text { NS } \\
<0.05\end{array}$ & $\begin{array}{l}77(8 \cdot 7) \\
75(15 \cdot 3) \\
78(12 \cdot 0)\end{array}$ \\
\hline & \multicolumn{3}{|c|}{ Average desaturation (\%) } & \multicolumn{3}{|c|}{ Maximum desaturation (\%) } \\
\hline $\begin{array}{l}\text { WAKE } \\
\text { NREM } \\
\text { REM }\end{array}$ & $\begin{array}{l}2(2 \cdot 8) \\
-6(6 \cdot 5) \\
-15(9 \cdot 2)\end{array}$ & $\begin{array}{l}<0.05 \\
<0.05 \\
<0.05\end{array}$ & $\begin{array}{l}+5(3 \cdot 4) \\
+2(4 \cdot 2) \\
-1(9 \cdot 2)\end{array}$ & $\begin{array}{l}-14(6 \cdot 6) \\
-21(9 \cdot 8) \\
-29(8 \cdot 1)\end{array}$ & $\begin{array}{l}\text { NS } \\
\text { NS } \\
<0.05\end{array}$ & $\begin{array}{l}-9(9 \cdot 2) \\
-11(15 \cdot 3) \\
-9(12 \cdot 3)\end{array}$ \\
\hline
\end{tabular}

( $2 \%$ to $23 \%$ ) higher at six months than during spontaneous ventilation at the onset of the study $(\mathrm{p}<0.05)$. This increase in $\mathrm{SaO}_{2}$ was seen during wakefulness and REM and nonREM sleep (table 2). The minimum $\mathrm{SaO}_{2}$ during sleep also increased during nasal intermittent positive pressure ventilation but this was significant only during REM sleep. A similar pattern was seen in the degree of desaturation from baseline (table 2). The mean $\mathrm{PtcCO}_{2}$ during sleep fell by $-2.7 \mathrm{kPa}(-1.3$ to $-5.1) ; p<0.05)$ and the mean increment in $\mathrm{PtcCO}_{2}$ during sleep was reduced by $-1 \cdot 7$ $(-0.3$ to $-3 \cdot 1 \mathrm{kPa} ; \mathrm{p}<0.05)$ during nasal intermittent positive pressure ventilation at six months compared with during spontaneous ventilation at the start of the study.

During spontaneous breathing patients had disturbed sleep with several arousals and long periods of wakefulness. During nasal intermittent positive pressure ventilation total sleep time increased by $72.5(21.5$ to 204.5$)$ minutes and sleep efficiency by $5 \%(-3 \%$ to $+30 \%)$, but there was no significant change in the number of arousals or in sleep latency (table 3). The increase in total sleep time was due to an increase in both REM and non-REM sleep; there was no clinically significant change in the proportion of REM to non-REM sleep.

Arterial blood gas tensions in the mid afternoon during spontaneous ventilation before the start of nasal intermittent positive pressure ventilation at six months and at one year are shown in figure 1 . The six month values in patients continuing to use the ventilator have been reported previously. ${ }^{4}$ At one year $\mathrm{PaO}_{2}$ was unchanged in the seven patients (median change compared with values before nasal

Table 3 Sleep quality during spontaneous breathing (SV) at start of and during nasal intermittent positive pressure ventilation (NIPPV) six months later. Values are means (SD)

\begin{tabular}{lccl}
\hline & $S V$ & NIPPV & $p$ \\
\hline Sleep latency (min) & $24(16)$ & $23(21)$ & NS \\
Total sleep time (min) & $269(66)$ & $349(74)$ & 0.02 \\
NREM total (min) & $207(44)$ & $273(47)$ & 0.04 \\
total (\%) & $78(9)$ & $79(11)$ & NS \\
stage II & $162(44)$ & $211(51)$ & NS \\
stage III and IV & $44(42)$ & $62(27)$ & NS \\
REM (min) & $62(31)$ & $75(47)$ & NS \\
(\%) & $22(9)$ & $20(11)$ & 0.02 \\
TST/TIB (\%) & $65(17)$ & $74(11)$ & 0.05 \\
Arousals (No) & $5.5(2 \cdot 1)$ & $4.6(3 \cdot 1)$ & NS \\
\hline
\end{tabular}

intermittent positive pressure ventilation $+0.6 \mathrm{kPa}(-0.5$ to +1.6$)$, NS) but all seven showed a further reduction in $\mathrm{PaCO}_{2}$ (by $-1 \cdot 7$ $\mathrm{kPa}(-2 \cdot 1$ to -0.6$), \mathrm{p}<0.5)$ and bicarbonate $(-6.3 \mathrm{mmol} / \mathrm{l}(-11$ to -4$), \mathrm{p}<0.05)$.

Quality of life was scored for breathlessness, mastery, mood, and fatigue (fig 3). Results were expressed as a percentage of maximum possible score, with an increase indicating an improvement. There was a trend towards improvement but no significant differences.

\section{Discussion}

This study shows that patients with severe chronic obstructive lung disease can be ventilated at home overnight for a prolonged period by nasal intermittent positive pressure ventilation. At the end of the one year study the seven patients continuing to have such ventilation all wanted to continue with it at home. Five of the seven patients had previously used domiciliary oxygen but they preferred nasal intermittent positive pressure ventilation, the main reasons being an enhanced sense of well being and improved quality of sleep.

Five patients did not take nasal intermittent positive pressure ventilation for the whole study period. Although those who dropped out initially tended to have fewer symptoms than the other patients, a further contributory factor may have been inadequate patient education and acclimatisation. Three of the patients who withdrew (cases 2,3, and 4) were discharged from hospital after having been taught how to use the equipment and they used it overnight for the first time at home without supervision. Subsequent patients remained in hospital until they were proficient and confident in the use of the equipment, which in most cases took two to three days. During the early phases of the trial the equipment failed on several occasions and patients lost confidence; this may have been a further reason why two patients (cases 2 and 3 ) withdrew. Though equipment failed for two other patients (cases 5 and 7), they had been alerted to this possibility and sought help when it occurred. The technical problems were subsequently resolved, but this highlights the necessity for a comprehensive breakdown and maintenance service in any programme of home ventilation. A lower drop out rate might be expected in future with better patient education and reliable equipment. 

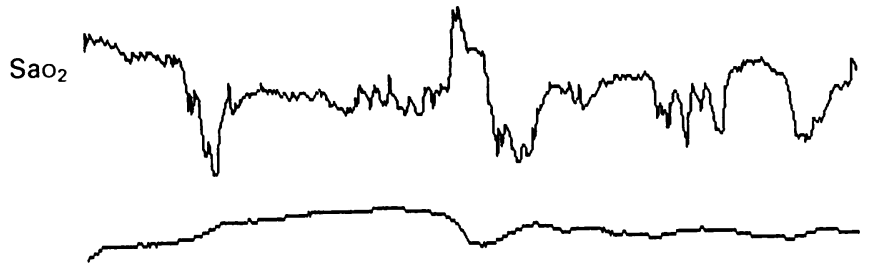
$100 \mathrm{~mm} \mathrm{Hg}$

$\mathrm{PtcCO}_{2}$

B NIPPV

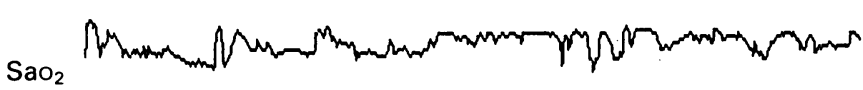

$\mathrm{PtccO}_{2}$

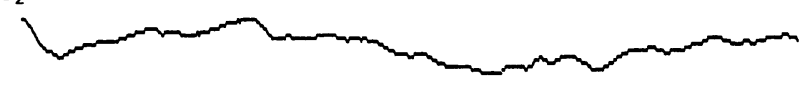

$40 \mathrm{~mm} \mathrm{Hg}$

Figure 2 Overnight oxygen saturation ( $\mathrm{SaO}_{2} \%$ ), and transcutaneous carbon dioxide tension $\left(\mathrm{PtcCO}_{2}\right)$ in case 11 during spontaneous breathing $(A)$ and nasal intermittent positive pressure ventilation $(N I P P V)(B)$. The increase in $\mathrm{SaO}_{2} \%$ in the middle of the night in $A$ occurred when the patient awoke to use a nebuliser.

Mean $\mathrm{SaO}_{2}$ and $\mathrm{PtcCO}_{2}$ overnight noticeably improved with nasal intermittent positive pressure ventilation in the patients continuing to take the treatment despite an increase in total sleep time during nasal intermittent positive pressure ventilation compared with spontaneous ventilation. This improvement was not simply a consequence of starting the night with better arterial blood gas tensions, as the increment in $\mathrm{PtcCO}_{2}$ and the magnitude of oxygen desaturation from waking values were reduced during all sleep stages. Although increased

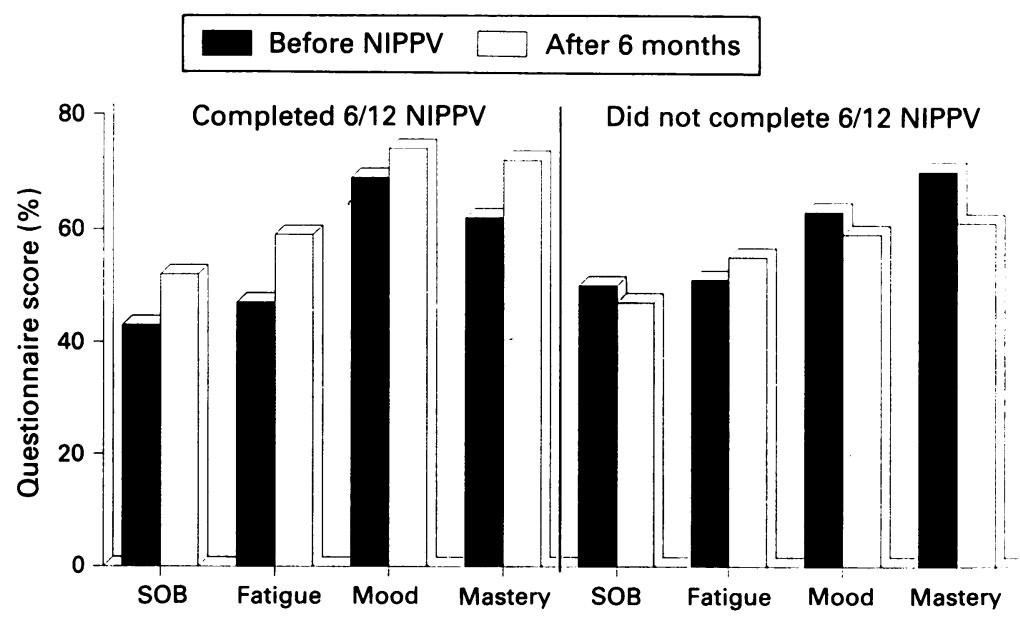

Figure 3 Percentage questionnaire scores in the patients using nasal intermittent positive pressure ventilation at six months (left) and those who had discontinued treatment (right). SOB-short of breath. alveolar ventilation is probably the predominant mechanism of the improved gas tensions during sleep, other factors may be important. Nasal intermittent positive pressure ventilation may improve matching of ventilation and perfusion by reducing or preventing the fall in functional residual capacity during sleep and causing re-expansion of atelectatic areas of lung.

Leakage around the mask and through the mouth may reduce ventilation, particularly during sleep. We used commercially available masks, which come in various different sizes, and an adequate seal between the mask and the patient's face was obtained in all cases. The problem of leakage from the mouth was diminished by using a chin strap, but most patients did not like this and had discarded it by the time of the follow up sleep study at six months. Leakage from the mouth was compensated for by using large minute volumes (median 23 (10-28) $1 / \mathrm{min}$ ), which resulted in high inflation pressures of $30-40 \mathrm{~cm} \mathrm{H}_{2} \mathrm{O}$ during wakefulness; with the onset of sleep these fell to $20-30 \mathrm{~cm} \mathrm{H}_{2} \mathrm{O}$ and blood gas tensions were successfully controlled. Additionally, patients were encouraged to have the inspiratory time as long as possible (median $1.3 \mathrm{~s}(0.8$ to 1.6$)$ and median inspiratory/expiratory ratio $1: 2 \cdot 0(1: 1 \cdot 7$ to $1: 4 \cdot 0)$ ) to minimise peak inflation pressures and promote gas exchange in diseased lung units with long time constants.

Improved quality of sleep was an important benefit of nasal intermittent positive pressure ventilation for the patients who continued treatment, and this was confirmed objectively by full polysomnography. Despite a night of acclimatisation, they still experienced moderate sleep disruption in the laboratory, but in their own homes most reported sleeping through the night or having only occasional arousals. The usual reasons for waking were a dry throat or painful gastric distension. The sleep studies were not performed in a random order as the study during nasal intermittent positive pressure ventilation always followed that during spontaneous breathing; the improvement in sleep duration during nasal intermittent positive pressure ventilation may therefore have been a consequence of familiarity with the sleep laboratory. However, a night of acclimatisation preceded the formal study on each occasion and the gap between the two studies was six months. Because of this long interval the changes seen are probably not simply the result of better acclimatisation. In addition, the changes documented in the laboratory confirm the subjective reports of the patients about the improved quality of sleep at home. This improvement may reflect a general improvement in clinical condition rather than an effect of nasal intermittent positive pressure ventilation itself.

Of the seven patients completing the trial, five had previously used oxygen at home and reported that sleep was subjectively better during nasal intermittent positive pressure ventilation. However, two of the patients who withdrew did so because they preferred oxygen therapy. The effect of oxygen on sleep quality is 
contentious, with some investigators showing an improvement ${ }^{9}$ and others no benefit. ${ }^{10}$ In neither of these studies was carbon dioxide tension measured but, although severe hypoxaemia $\left(\mathrm{SaO}_{2}<70 \%\right)$ may not cause arousal in humans, ${ }^{11}$ acute hypercapnia with an increase in $\mathrm{PaCO}_{2}$ of 6 to $15 \mathrm{~mm} \mathrm{Hg}$ is a reliable and powerful arousal stimulus. ${ }^{12}$ Therefore the improved sleep during nasal intermittent positive pressure ventilation may reflect the reduced $\mathrm{PtCCO}_{2}$, but other factors may also be important. The three patients who withdrew had difficulties sleeping with the equipment and had had relatively undisturbed sleep before starting positive pressure ventilation. Disturbed sleep during spontaneous breathing seems to be an important requirement for a successful outcome.

The previously reported improvements in $\mathrm{PaCO}_{2}$ and bicarbonate ion concentration were maintained and, in most patients, improved further at one year. These differences might have been larger in cases 8 and 11 if the patients had not had a period of in hospital nasal intermittent positive pressure ventilation before being recruited to the study. These data suggest that the full benefit of domiciliary treatment develops over a relatively long period.

Although objective measurement of quality of life did not show significant changes, it was no worse during nasal intermittent positive pressure ventilation. Five patients thought that their lives had been transformed, which was substantiated by changes in lifestyle. Three patients who had been effectively housebound or in hospital for at least a year were able to leave the house to do their own shopping, etc. One patient, retired on medical grounds for three years, was seeking part time employment. In the study of negative pressure ventilation by Zibrak et al lack of improvement was an important reason for withdrawal from the study. ${ }^{13}$ The desire of seven patients at one year to continue using nasal intermittent positive pressure ventilation during sleep suggests useful symptomatic benefit.

This was an uncontrolled study and patients received more personal attention than is normal in busy clinical practice; a large placebo effect cannot be discounted. Long term oxygen therapy remains the preferred treatment in patients with respiratory failure due to chronic obstructive lung disease, having been shown to improve survival in two large clinical trials. ${ }^{14} 15$ In practice, however, patients are often poorly compliant, ${ }^{1617}$ the main reason being the restriction placed on lifestyle by the need for 15 hours of oxygen a day. Compliance with nasal intermittent positive pressure ventilation, which needs to be used only during sleep, may be better. Our study suggests, however, that acceptability may be greater in patients with more severe symptoms, as is the case with long term oxygen therapy.

Hypercapnia seems to be associated with a higher mortality and diminished response to long term oxygen therapy. In the trial by the Medical Research Council survival benefit from oxygen was not seen until after 500 days of treatment had elapsed, the best predictor of death during this period being a combination of polycythaemia and hypercapnia. ${ }^{14}$ In another study, in which benefit from long term oxygen therapy was apparent immediately, 29 out of 57 patients who were hypercapnic on entry died during the study compared with only 3 out of 15 who were normocapnic. ${ }^{18} \mathrm{~A}$ reduction in hypercapnia may therefore improve survival. However Robert et al, giving patients with chronic obstructive lung disease positive pressure ventilation via a tracheostomy, found no difference in survival compared with the results in patients given oxygen in other studies. ${ }^{19}$ The effect of nocturnal ventilation on $\mathrm{PaCO}_{2}$ was not recorded and a tracheostomy may be associated with a significant morbidity and mortality. ${ }^{20}$ The improvements in oxygenation in our study during nasal intermittent positive pressure ventilation and during the day were less than those seen with long term oxygen therapy. The lower $\mathrm{PaO}_{2}$ may have an adverse effect on survival; although oxygen could be added through an integral port in the mask, this would add to the cost and complexity of home treatment.

Continuous positive airway pressure is cheaper and easier to administer than nasal intermittent positive pressure ventilation and was effective in controlling nocturnal desaturation in a patient with severe obesity hypoventilation syndrome. ${ }^{21}$ However, the effect on daytime or night time $\mathrm{PaCO}_{2}$ was not evaluated, and when continuous positive airway pressure was used in patients with kyphoscoliosis during sleep it was ineffective in controlling hypercapnia, except in two patients with predominantly upper airway obstruction. ${ }^{2}$ Continuous positive airway pressure is therefore unlikely to be effective in patients with chronic obstructive lung disease unless they have predominantly upper airway obstruction.

Thus our study shows that nasal intermittent positive pressure ventilation can be used effectively in some patients with chronic obstructive lung disease but its further place in the management of chronic respiratory failure due to such disease can be determined only by a large scale randomised comparison with long term oxygen therapy. The advantage of a treatment administered only at night that causes symptomatic benefit during the day may prove to be more important to patients than any effects on survival. Well motivated patients are most likely to benefit, and adequate education and acclimatisation to the technique are essential. Although it cannot currently be recommended as first line treatment for hypercapnic patients with chronic obstructive lung disease, a trial of nasal intermittent positive pressure ventilation should be considered in those whose condition is deteriorating despite oxygen therapy or who are intolerant of it.

MWE was supported by the Royal Brompton Hospital Clinical Research Committee. The ventilators were purchased with a grant from the Department of Health. The technical help of Messrs Derek Cramer and Simon Ward and the staff of the Lung Function Laboratory at the Royal Brompton Hospital is gratefully acknowledged. 
1 Ellis ER, Bye PTB, Bruderer JW, Sullivan CE. Treatment of respiratory failure during sleep in patients with neuromuscular disease. Am Rev Respir Dis 1987;135: neuromusc-52.

2 Ellis ER, Grunstein RR, Chan S, Bye PTB, Sullivan CE. Noninvasive ventilatory support during sleep improves respiratory failure in kyphoscoliosis. Chest 1988;94:811-5.

3 Carroll N, Branthwaite MA. Control of nocturnal hypoventilation by nasal intermittent positive pressure ventilaventilation by nasal intermitten

4 Elliott MW, Mulvey DA, Moxham J, Green M, Branthwaite MA. Domiciliary nocturnal nasal intermittent positive pressure ventilation in COPD: mechanisms underlying changes in arterial blood gas tensions. Eur Respir J 1991 4:1044-5.

5 Sériès F, Cormier Y, La Forge J. Changes in day and night time oxygenation with protryptiline in patients with time oxygenation with protryptiline in patients with
chronic obstructive lung disease. Thorax 1989;44:275-9.

6 Carroll N, Parker RA, Branthwaite MA. The use of protryptiline for respiratory failure in patients with chronic airflow limitation. Eur Respir J 1990;3:746-51.

7 Rechtschaffen A, Kales A, eds. $A$ manual of standard terminology, techniques and scoring system for sleep stages of human subjects. Bethesda, Maryland: National Institute of Neurological Disease and Blindness. (NIH publication No 204.)

8 Guyatt GH, Berman LB, Townsend M, Pugsley SO Chambers LW. A measure of quality of life for clinical trials in chronic lung disease. Thorax 1987;42:773-8.

9 Calverley PMA, Brezinova V, Douglas NJ, Catterall JR, Flenley DC. The effect of oxygenation on sleep quality in chronic bronchitis and emphysema. Am Rev Respir Dis 1982;126:206-10.

10 Fleetham JA, West P, Mezon B, Conway W, Roth T, Kryger $M$. Sleep, arousals, and oxygen desaturation in COPD Am Rev Respir Dis 1982;126:429-33.

11 Berthon-Jones $M$, Sullivan CE. Ventilatory and arousal responses to hypoxia in sleeping humans. Am Rev Respir Dis 1982;125:632-9.
12 Hedemark L, Kronenberg $R$. Ventilatory responses to hypoxia and $\mathrm{CO}_{2}$ during natural and flurazepam induced sleep in normal adults. Am Rev Respir Dis 1981;123:190 (abstract).

13 Zibrak JD, Hill NS, Federman EC, Kwa SL, O'Donnell C. Evaluation of intermittent long term negative-pressure ventilation in patients with severe COPD. Am Rev Respir Dis 1988;138:1515-8.

14 Medical Research Council Working Party Report. Long term domiciliary oxygen therapy in chronic hypoxic cor pulmonale complicating chronic bronchitis and emphysema. Lancet 1981;i:681-5.

15 Nocturnal Oxygen Therapy Trial Group. Continuous or nocturnal oxygen therapy in hypoxemic chronic obstructive lung disease, a clinical trial. Ann Intern Med 1980; 93:391-8.

16 Baudouin SV, Waterhouse JC, Tahtamouni T, Smith JA, Baxter J, Howard P. Long term domiciliary oxygen treatment for chronic respiratory failure reviewed. Thorax 1990;45:195-8.

17 Walshaw MJ, Lim R, Evans CC, Hind CRK. Factors influencing the compliance of patient using oxygen concentrators for long-term home oxygen therapy. Respir Med 1990;84:331-3.

18 Cooper CB, Waterhouse J, Howard P. Twelve year clinical study of patients with hypoxic cor pulmonale given long term domiciliary oxygen therapy. Thorax 1987;42:105-10.

19 Robert D, Gerard M, Leger P, Buffat J, Jennequin J, Holzapfel L, et al. Domiciliary ventilation by tracheostomy for chronic respiratory failure. Rev Fr Mal Resp 1983;11:923-6.

20 Stauffer JL, Olson DE, Petty TL. Complications and consequences of endotracheal intubation and tracheostomy. Am J Med 1981;70:65-75.

21 Sériès F, Cormier Y, La Forge J. Non-apnoeic REM sleep induced nocturnal oxygen desaturation treated by nasal continuous positive airway pressure. Thorax 1989;44: 521-2. 